\title{
BACKWARD VOLUME CONTRACTION FOR ENDOMORPHISMS WITH EVENTUAL VOLUME EXPANSION
}

\author{
JOSÉ F. ALVES, VILTON PINHEIRO, AND ARMANDO CASTRO
}

\begin{abstract}
We consider smooth maps on compact Riemannian manifolds. We prove that under some mild condition of eventual volume expansion Lebesgue almost everywhere we have uniform backward volume contraction on every pre-orbit for Lebesgue almost every point.
\end{abstract}

\section{Statement of Results}

Let $M$ be a compact Riemannian manifold and let Leb be a volume form on $M$ that we call Lebesgue measure. We take $f: M \rightarrow M$ any smooth map. Let $0<a_{1} \leq a_{2} \leq a_{3} \leq \ldots$ be a sequence converging to infinity. We define

$$
h(x)=\min \left\{n>0:\left|\operatorname{det} D f^{n}(x)\right| \geq a_{n}\right\},
$$

if this minimum exists, and $h(x)=\infty$, otherwise. For $n \geq 1$, we take

$$
\Gamma_{n}=\{x \in M: h(x) \geq n\} .
$$

Theorem 1.1. Assume that $h \in L^{p}(\mathrm{Leb})$, for some $p>3$, and take $\gamma<(p-3) /(p-1)$. Choose any sequence $0<b_{1} \leq b_{2} \leq b_{3} \leq \ldots$ such that $b_{k} b_{n} \geq b_{k+n}$ for every $k, n \in \mathbb{N}$, and assume that there is $n_{0} \in \mathbb{N}$ such that $b_{n} \leq \min \left\{a_{n}, \operatorname{Leb}\left(\Gamma_{n}\right)^{-\gamma}\right\}$ for every $n \geq n_{0}$. Then, for Leb almost every $x \in M$, there exists $C_{x}>0$ such that $\left|\operatorname{det} D f^{n}(y)\right|>C_{x} b_{n}$ for every $y \in f^{-n}(x)$.

We say that $f: M \rightarrow M$ is eventually volume expanding if there exists $\lambda>0$ such that for Lebesgue almost every $x \in M$

$$
\sup _{n \geq 1} \frac{1}{n} \log \left|\operatorname{det} D f^{n}(x)\right|>\lambda .
$$

Let $h$ and $\Gamma_{n}$ be defined as in (11) and (2), associated to the sequence $a_{n}=e^{\lambda n}$.

Date: June 4, 2018.

Work carried out at the Federal University of Bahia. Partially supported by FCT through CMUP and UFBA. 
Corollary 1.2. If $f$ is eventually volume expanding, then for Lebesgue almost every point $x \in M$ there are $C_{x}>0$ and $\sigma_{n} \rightarrow \infty$ such that $\left|\operatorname{det} D f^{n}(y)\right|>C_{x} \sigma_{n}$ for every $y \in f^{-n}(x)$. Moreover, given $\alpha>0$ there is $\beta>0$ such that

(1) if $\operatorname{Leb}\left(\Gamma_{n}\right) \leq \mathcal{O}\left(e^{-\alpha n}\right)$, then we may take $\sigma_{n} \geq e^{\beta n}$;

(2) if $\operatorname{Leb}\left(\Gamma_{n}\right) \leq \mathcal{O}\left(e^{-\alpha n^{\tau}}\right)$ for some $\tau>0$, then we may take $\sigma_{n} \geq e^{\beta n^{\tau}}$

(3) if $\operatorname{Leb}\left(\Gamma_{n}\right) \leq \mathcal{O}\left(n^{-\alpha}\right)$ and $\alpha>2$, then we may take $\sigma_{n} \geq n^{\beta}$.

Specific rates will be obtained in Section 4 for some eventually volume expanding endomorphisms. In particular, non-uniformly expanding maps such as quadratic maps and Viana maps will be considered.

\section{Conchtenated Collections}

Let $\left(U_{n}\right)_{n}$ be a collection of measurable subsets of $M$ whose union covers a full Lebesgue measure subset of $M$. We say that $\left(U_{n}\right)_{n}$ is a concatenated collection if:

$$
x \in U_{n} \quad \text { and } \quad f^{n}(x) \in U_{m} \quad \Rightarrow \quad x \in U_{n+m} .
$$

Given $x \in \bigcup_{n>1} U_{n}$, we define $u(x)$ as the minimum $n \in \mathbb{N}$ for which $x \in U_{n}$. Note that by definition we have $x \in U_{u(x)}$. We define the chain generated by $x \in \bigcup_{n \geq 1} U_{n}$ as $C(x)=\left\{x, f(x), \ldots, f^{u(x)-1}(x)\right\}$.

Lemma 2.1. Let $\left(U_{n}\right)_{n}$ be a concatenated collection. If

$$
\sum_{n \geq 1} \sum_{j=0}^{n-1} \operatorname{Leb}\left(f^{j}\left(u^{-1}(n)\right)\right)<\infty,
$$

then we have $\sup \left\{u(y): y \in \bigcup_{n \geq 1} U_{n}\right.$ and $\left.x \in C(y)\right\}<\infty$ for Lebesgue almost every $x \in M$.

Proof. Assume that for a given $x \in M$ there exists an infinite number of chains $C_{j}=\left\{y_{j}, f\left(y_{j}\right), \ldots, f^{s_{j}-1}\left(y_{j}\right)\right\}, j \geq 1$, containing $x$ with $s_{j} \rightarrow \infty$. For each $j \geq 1$ let $1 \leq r_{j}<s_{j}$ be such that $x=$ $f^{r_{j}}\left(y_{j}\right)$. First we verify that $\lim r_{j}=\infty$. If not, then replacing by a subsequence, we may assume that there is $N>0$ such that $r_{j}<N$ for every $j \geq 1$. This implies that $y_{j} \in \bigcup_{i=1}^{N} f^{-i}(x)$ for every $j \geq 1$. Since $\#\left(\bigcup_{i=1}^{N} f^{-i}(x)\right)<\infty$ and the number of chains is infinite, we have a contradiction. Since $r_{j} \rightarrow \infty$ and $x=f^{r_{j}}\left(y_{j}\right) \in$ $f^{r_{j}}\left(u^{-1}\left(s_{j}\right)\right)$, then we have $x \in \bigcup_{n \geq k} \bigcup_{j=0}^{n-1} f^{j}\left(u^{-1}(n)\right)$ for every $k \geq 1$. Since we are assuming $\sum_{n \geq 1} \sum_{j=0}^{n-1} \operatorname{Leb}\left(f^{j}\left(u^{-1}(n)\right)\right)<\infty$, we have Leb $\left(\bigcup_{n \geq k} \bigcup_{j=0}^{n-1} f^{j}\left(u^{-1}(n)\right)\right) \rightarrow 0$, when $k \rightarrow \infty$. This completes the proof of Lemma 2.1. 
Lemma 2.2. Let $\left(U_{n}\right)_{n}$ be a concatenated collection. If

$$
\sup \left\{u(y): y \in \cup_{n \geq 1} U_{n} \text { and } x \in C(y)\right\} \leq N,
$$

then $f^{-n}(x) \subset U_{n} \cup \cdots \cup U_{n+N}$ for all $n \geq 1$.

Proof. Assume that $\sup \left\{u(y): y \in \cup_{n \geq 1} U_{n}\right.$ and $\left.x \in C(y)\right\} \leq N$, and take $z \in f^{-n}(x)$. Let $z_{j}=f^{j}(z)$ for each $j \geq 0$. We distinguish the cases $x \in C(z)$ and $x \notin C(z)$. If $x \in C(z)$, then $n \leq u(z) \leq n+N$. Hence $z \in U_{u(z)} \subset U_{n} \cup \cdots \cup U_{n+N}$. If $x \notin C(z)$, then letting $u_{0}=u(z)$ we must have $u_{0}<n$. Let $u_{1}=u\left(z_{u_{0}}\right)$. If $u_{0}+u_{1}<n$ we take $u_{2}=u\left(z_{u_{0}+u_{1}}\right)$. We proceed in this way until we find the first $s \leq n$ such that $n \leq u_{0}+\cdots+u_{s}$. Note that $u_{s}=u\left(z_{u_{0}+\cdots+u_{s-1}}\right)$, and by the choice of $s$ we must have $x \in C\left(z_{u_{0}+\cdots+u_{s-1}}\right)$. Our assumption implies that $u\left(z_{u_{0}+\cdots+u_{s-1}}\right) \leq N$, and so $u_{0}+\cdots+u_{s} \leq n+N$. By construction we have

$$
\begin{aligned}
& z \in U_{u_{0}} \\
& f^{u_{0}}(z)=z_{u_{0}} \in U_{u_{1}} \\
& f^{u_{0}+u_{1}}(z)=z_{u_{0}+u_{1}} \in U_{u_{2}} \\
& f^{u_{0}+\cdots u_{s-1}}(z)=z_{u_{0}+\cdots u_{s-1}} \in U_{u_{s}}
\end{aligned}
$$

By the definition of a concatenated collection we conclude that $z \in$ $U_{u_{0}+u_{1}+\cdots+u_{s}}$.

\section{Proofs of MAIN ReSults}

Let us now prove Theorem 1.2. Suppose that $h \in L^{p}$ (Leb), for some $p>3$. This implies that $\sum_{n \geq 1} n^{p} \operatorname{Leb}\left(h^{-1}(n)\right)<\infty$, and so there exists some constant $K>0$ such that

$$
\operatorname{Leb}\left(h^{-1}(n)\right) \leq K n^{-p}, \quad \text { for every } n \geq 1 .
$$

Now, taking $0<\gamma<(p-3) /(p-1)$ we have for some $K^{\prime}>0$

$$
\sum_{n=1}^{\infty} n\left(\sum_{k=n}^{\infty} \operatorname{Leb}\left(h^{-1}(k)\right)\right)^{1-\gamma} \leq \sum_{n=1}^{\infty} n\left(K^{\prime} / n^{p-1}\right)^{1-\gamma}<\infty .
$$

Defining

$$
U_{n}=\left\{x \in M:\left|\operatorname{det} D f^{n}(x)\right| \geq b_{n}\right\},
$$

then we have that $\left(U_{n}\right)_{n}$ is a concatenated collection with respect to the Lebesgue measure. Moreover, setting

$$
U_{n}^{*}=U_{n} \backslash\left(U_{1} \cup \ldots \cup U_{n-1}\right)
$$

one has $U_{n}^{*} \subset \bigcup_{m \geq n} h^{-1}(m)$, for otherwise there would be $x \in U_{n}^{*} \cap$ $h^{-1}(m)$ with $m<n$, and so $a_{m} \geq b_{m}>\left|\operatorname{det} D f^{m}(x)\right| \geq a_{m}$, which is 
not possible. As $\left|\operatorname{det} D f^{j}(x)\right|<b_{j}$ for every $x \in U_{n}^{*}$ and $j<n$, we get $\operatorname{Leb}\left(f^{j}\left(U_{n}^{*}\right)\right) \leq b_{j} \operatorname{Leb}\left(U_{n}^{*}\right)$ for each $j<n$. Hence

$$
\begin{aligned}
\sum_{n=n_{0}+1}^{\infty} \sum_{j=0}^{n-1} \operatorname{Leb}\left(f^{j}\left(U_{n}^{*}\right)\right) & \leq \sum_{n=n_{0}+1}^{\infty} \sum_{j=0}^{n-1} b_{j} \operatorname{Leb}\left(U_{n}^{*}\right) \\
& \leq \sum_{n=n_{0}+1}^{\infty} \sum_{j=0}^{n_{0}-1} b_{j} \operatorname{Leb}\left(U_{n}^{*}\right)+\sum_{n=n_{0}+1}^{\infty} \sum_{j=n_{0}}^{n-1} b_{j} \operatorname{Leb}\left(U_{n}^{*}\right) \\
& \leq \sum_{j=0}^{n_{0}-1} b_{j}+\sum_{n=n_{0}+1}^{\infty} \sum_{j=n_{0}}^{n-1} b_{j} \operatorname{Leb}\left(U_{n}^{*}\right)
\end{aligned}
$$

Now we just have to check that the last term in the sum above is finite. Indeed,

$$
\begin{aligned}
\sum_{n=n_{0}+1}^{\infty} \sum_{j=n_{0}}^{n-1} b_{j} \operatorname{Leb}\left(U_{n}^{*}\right) & \leq \sum_{n=n_{0}+1}^{\infty} \sum_{j=n_{0}}^{n-1} b_{j} \sum_{k=n}^{\infty} \operatorname{Leb}\left(h^{-1}(k)\right) \\
& \leq \sum_{n=n_{0}+1}^{\infty} n b_{n} \sum_{k=n}^{\infty} \operatorname{Leb}\left(h^{-1}(k)\right) \\
& \leq \sum_{n=n_{0}+1}^{\infty} n\left(\sum_{k=n}^{\infty} \operatorname{Leb}\left(h^{-1}(k)\right)^{-\gamma} \sum_{k=n}^{\infty} \operatorname{Leb}\left(h^{-1}(k)\right)\right. \\
& =\sum_{n=n_{0}+1}^{\infty} n\left(\sum_{k=n}^{\infty} \operatorname{Leb}\left(h^{-1}(k)\right)\right)^{1-\gamma}<\infty
\end{aligned}
$$

Applying Lemmas 2.1] and 2.2, we get for each generic point $x \in M$ a positive integer number $N_{x}$ such that if $y \in f^{-n}(x)$ then $y \in U_{n+s}$ for some $0 \leq s \leq N_{x}$. Therefore, $\left|\operatorname{det} D f^{n+s}(y)\right|>b_{n+s} \geq b_{n}$. Then, taking $C_{x}=K^{-N_{x}}$, where $K=\sup \{|\operatorname{det} D f(z)|: z \in M\}$, we obtain the conclusion of Theorem 1.1.

$$
\left|\operatorname{det} D f^{n}(y)\right|=\frac{\left|\operatorname{det} D f^{n+s}(y)\right|}{\left|\operatorname{det} D f^{s}(x)\right|}>C_{x} b_{n} .
$$

Now we explain how we use Theorem 1.1 to prove Corollary 1.2 Recall that in Corollary 1.2 we have $a_{n}=e^{\lambda n}$ for each $n \in \mathbb{N}$. Assume first that $\operatorname{Leb}\left(\Gamma_{n}\right) \leq \mathcal{O}\left(e^{-c^{\prime} n}\right)$ for some $c^{\prime}>0$. Then it is possible to choose $c>0$ such that $b_{n}=e^{c n}$, for $n \geq n_{0}$. The other two cases are obtained under similar considerations. 


\section{EXAMPLES: NON-UNIFORMLY EXPANDING MAPS}

An important class of dynamical systems where we can immediately apply our results are the non-uniformly expanding dynamical maps introduced in 2. As particular examples of this kind of systems we present below quadratic maps and the higher dimensional Viana maps. Quadratic maps. Let $f_{a}:[-1,1] \rightarrow[-1,1]$ be given by $f_{a}(x)=1-a x^{2}$, for $0<a \leq 2$. Results in [3, 8] give that for a positive Lebesgue measure set of parameters $f_{a}$ in non-uniformly expanding. Ongoing work [5] gives that for a positive Lebesgue measure set of parameters there are $C, c>0$ such that $\operatorname{Leb}\left(\Gamma_{n}\right) \leq C e^{-c n}$ for every $n \geq 1$.

Thus, it follows from Corollary 1.2 that we may find $\beta>0$ such for Lebesgue almost every $x \in I$ there is $C_{x}>0$ such that $\left|\left(f^{n}\right)^{\prime}(y)\right|>$ $C_{x} e^{\beta n}$ for every $y \in f^{-n}(x)$.

Viana maps. Let $a_{0} \in(1,2)$ be such that the critical point $x=0$ is pre-periodic for the quadratic map $Q(x)=a_{0}-x^{2}$. Let $S^{1}=\mathbb{R} / \mathbb{Z}$ and $b: S^{1} \rightarrow \mathbb{R}$ given by $b(s)=\sin (2 \pi s)$. For fixed small $\alpha>0$, consider the map $\hat{f}$ from $S^{1} \times \mathbb{R}$ into itself given by $\hat{f}(s, x)=(\hat{g}(s), \hat{q}(s, x))$, where $\hat{q}(s, x)=a(s)-x^{2}$ with $a(s)=a_{0}+\alpha b(s)$, and $\hat{g}$ is the uniformly expanding map of $S^{1}$ defined by $\hat{g}(s)=d s(\bmod \mathbb{Z})$ for some integer $d \geq 2$. For $\alpha>0$ small enough there is an interval $I \subset(-2,2)$ for which $\hat{f}\left(S^{1} \times I\right)$ is contained in the interior of $S^{1} \times I$. Thus, any map $f$ sufficiently close to $\hat{f}$ in the $C^{0}$ topology has $S^{1} \times I$ as a forward invariant region. Moreover, there are $C, c>0$ such that $\operatorname{Leb}\left(\Gamma_{n}\right) \leq$ $C e^{-c \sqrt{n}}$ for every $n \geq 1$; see [1, 4, 9.

Thus, it follows from Corollary 1.2 that we may find $\beta>0$ such for Lebesgue almost every $X \in S^{1} \times I$ there is a constant $C_{X}>0$ such that $\left|\operatorname{det} D f^{n}(Y)\right|>C_{X} e^{\beta \sqrt{n}}$ for every $Y \in f^{-n}(X)$.

\section{REFERENCES}

[1] J. F. Alves, V. Araújo, Random perturbations of nonuniformly expanding maps, Astérisque 286 (2003), 25-62.

[2] J. F. Alves, C. Bonatti, M. Viana, SRB measures for partially hyperbolic systems whose central direction is mostly expanding, Invent. Math. 140 (2000), 351-398.

[3] M. Benedicks, L. Carleson, On iterations of $1-a x^{2}$ on $(-1,1)$, Ann. Math. 122 (1985), 1-25.

[4] J. Buzzi, O. Sester, M. Tsujii, Weakly expanding skew-products of quadratic maps, Ergodic Theory Dynam. Systems 23 (2003), no. 5, 1401-1414

[5] J. Freitas, in preparation.

[6] A. Castro, Backward inducing and exponential decay of correlations for partially hyperbolic attractors, Israel J. Math. 130 (2002), 29-75. 
[7] A. Castro, Fast mixing for attractors with mostly contracting central direction, Ergodic Th. Dynam. \& Syst., to appear.

[8] M. Jakobson, Absolutely continuous invariant measures for one-parameter families of one-dimensional maps, Comm. Math. Phys. 81 (1981), 39-88.

[9] M. Viana, Multidimensional non-hyperbolic attractors, Publ. Math. IHES 85 (1997), 63-96.

Departamento de Matemática Pura, Faculdade de Ciências do Porto, Rua do Campo Alegre 687, 4169-007 Porto, Portugal

E-mail address: jfalves@fc.up.pt

Departamento de Matemática, Universidade Federal da Bahia, Av. Ademar de Barros s/n, 40170-110 SAlvador, Brazil.

E-mail address: viltonj@ufba.br

Departamento de Matemática, Universidade Federal da Bahia, Av. Ademar de Barros s/n, 40170-110 SAlvador, Brazil.

E-mail address: armando@im.ufba.br 